\title{
BMJ Open Consolidation chemotherapy in postmolar low-risk gestational trophoblastic neoplasia: a systematic review protocol
}

\author{
Mariza Branco-Silva (D) , ${ }^{1}$ Izildinha Maesta, ${ }^{2,3}$ Kevin Elias, ${ }^{4}$ Ross Stuart Berkowitz, ${ }^{4}$ \\ Joelcio Francisco Abbade, ${ }^{2}$ Neil S Horowitz ${ }^{4}$
}

To cite: Branco-Silva M,

Maesta I, Elias K, et al. Consolidation chemotherapy in postmolar low-risk gestational trophoblastic neoplasia: a systematic review protocol. BMJ Open 2022;12:e059484. doi:10.1136/ bmjopen-2021-059484

- Prepublication history and additional supplemental material for this paper are available online. To view these files, please visit the journal online (http://dx.doi.org/10.1136/ bmjopen-2021-059484).

Received 22 November 2021 Accepted 04 February 2022

Check for updates

(C) Author(s) (or their employer(s)) 2022. Re-use permitted under CC BY-NC. No commercial re-use. See rights and permissions. Published by BMJ.

For numbered affiliations see end of article.

Correspondence to Ms Mariza Branco-Silva; marizab@yahoo.com

\section{ABSTRACT}

Introduction Current evidence remains insufficient to strongly demonstrate the benefits of consolidation chemotherapy to all women with low-risk gestational trophoblastic neoplasia (GTN). This protocol outlines a systematic review to investigate whether consolidation chemotherapy is necessary for all patients with postmolar low-risk GTN after human chorionic gonadotropin normalisation with first-line single-agent chemotherapy. Methods and analysis A search string will be used to search the PubMed (MEDLINE), EMBASE, Web of Sciences, Scopus, LILACS and Cochrane Central Register of Controlled Trials databases. Articles will be screened at the title and abstract level, and then at the full article level by two independent reviewers using inclusion/ exclusion criteria. Randomised and non-randomised study designs will be included, while case studies, commentaries, editorials, review articles, animal studies, basic science studies and cross-sectional studies, as well as studies not reporting relapse/recurrence rates and/ or whether consolidation chemotherapy was delivered will be excluded. There will be no restrictions on date of publication, geographical location, study setting, or language of publication. The primary outcome is rate of recurrence/relapse. The assessments of randomised controlled trials will be performed using the risk of bias tool from the Cochrane Collaboration. Non-randomised studies will be assessed using the Newcastle-0ttawa scale. The quality of evidence will be assessed using the Grading quality of evidence and strength of recommendations (Grades of Recommendations, Assessment, Development and Evaluation) guidelines. Ethics and dissemination No formal ethical approval is required as all data collected will be secondary data and analysed anonymously. Results will be disseminated through a peer-reviewed publication and at scientific events.

PROSPERO registration number CRD42020164822.

\section{INTRODUCTION}

Gestational trophoblastic neoplasia (GTN) refers to a group of malignant lesions that arise from placental villous and extravillous trophoblast and may follow a hydatidiform mole or a nonmolar pregnancy. ${ }^{12}$ Based
Strengths and limitations of this study

- The number of randomised controlled trials found is expected to be small as this type of study usually requires a large sample size not easily available in the context of rare cancers such as gestational trophoblastic neoplasia (GTN).

- Differences in GTN diagnostic and classification criteria, as well as in the definition of relapse, may confound comparisons.

- The rigorous and transparent study design will possibly reduce the risk of biases.

- Grading the quality of the evidence will provide confidence in the effect estimates.

on GTN classification and stage, treatment consists of either single agent therapy with methotrexate (MTX) or Actinomycin D for low-risk disease, or multiagent therapy for high-risk disease. ${ }^{3}$

Chemotherapy should be continued until complete remission is achieved, with a normalised serum human chorionic gonadotropin (hCG) level. ${ }^{4}$ However, tumour resistance or relapse after first-line chemotherapy has been reported in approximately $25 \%$ of women with low-risk GTN. ${ }^{5}$ To eradicate the remaining tumour cells, consolidation therapy with the last effective agent(s) has been recommended to prevent relapse. ${ }^{4}$

Few studies have addressed consolidation therapy for low-risk GTN. Yang et al identified less than two courses of consolidation chemotherapy as a risk factor for relapsed disease ${ }^{6}$ while Sun et al published results suggesting the opposite. ${ }^{7}$ This is likely to be due to the fact that besides using different definitions for GTN and relapse, those studies combined patients with postmolar GTN with patients with post-delivery GTN, and patients normalising on MTX with patients who had progressive disease on MTX. 
Lybol et al retrospectively compared relapse rates following two (The Netherlands) or three (UK) MTX consolidation courses in women completing MTX therapy for low-risk GTN. They found a $4 \%$ relapse rate after three consolidation courses of MTX alternating with folinic acid in contrast to an $8.3 \%$ relapse rate following two consolidation courses, suggesting that three courses of consolidation chemotherapy is preferable to two in order to decrease the risk of low-risk GTN relapse. Nonetheless, differences between the Netherlands and the UK regarding the scoring systems employed in defining low-risk disease GTN, and in hCG measurement methods might have influenced the composition of the patient groups studied and the relapse rates observed. ${ }^{8}$

Couder et al retrospectively analysed the predictive factors of relapse in a homogeneous population with FIGO-2000-defined low risk GTN that received two consolidation courses after hCG normalisation with MTX alone. They found a relapse rate of $5.7 \%$, consistent with the rate reported in previous series where the FIGO scoring system was not uniformly used. Additionally, they demonstrated that postpartum low-risk GTN and patients who need more than four courses of MTX for normalisation are at a higher risk of relapse than other lowrisk patients (8.66-fold and 6.7-fold higher relapse risk, respectively). According to these authors, these findings suggest different biological behaviours among low-risk GTN patients and support previously reported relapse rates as low as $3 \%$ without any consolidation. ${ }^{9}$

Taken together, these data indicate that current evidence on the benefits of consolidation chemotherapy to all women with low-risk GTN remains insufficiently strong. Thus, this review aims at investigating whether consolidation chemotherapy is necessary for all patients with postmolar low-risk GTN after hCG normalisation with first-line single-agent chemotherapy. To this end, the proposed review will determine patient, disease and treatment characteristics of the women with postmolar lowrisk GTN included in each of the studies of interest; and assess the relationship of relapse rate with these characteristics, use of consolidation chemotherapy, and number of consolidation chemotherapy cycles used (if any).

\section{Review question}

The components of population, intervention, comparator, outcome and time frame are as follows:

Population: women with postmolar low-risk GTN who achieved remission (hCG normalisation) with singleagent chemotherapy.

Intervention: Consolidation chemotherapy after hCG normalisation with first-line single-agent chemotherapy.

Comparator: No consolidation treatment or placebo after hCG normalisation with first-line single-agent chemotherapy.

Outcome: The primary outcome is rate of recurrence/ relapse. Due to possible variation in disease definitions over time, definitions of outcomes will be extracted as reported in individual studies. Secondary outcomes will include consolidation chemotherapy toxicity and time to relapse/recurrence.

Time frame: up to 1 year of follow-up after hCG normalisation with first-line single-agent chemotherapy.

This protocol outlines the procedures for a systematic literature review intended to answer the question: Is consolidation chemotherapy necessary for all patients with postmolar low-risk GTN to prevent recurrence after hCG normalisation with first-line single-agent chemotherapy?

\section{METHODS AND ANALYSIS}

This protocol was designed according to PRISMA (Preferred Reporting Items for Systematic Reviews and Meta-Analysis Protocols) guidelines ${ }^{10}$ (checklist in online supplemental file 1).

\section{Eligibility criteria}

As information on consolidation therapy for postmolar low-risk GTN is limited, the following randomised and non-randomised study designs will be included: randomised and quasi-randomised trials (including cluster and cross-over trials), non-randomised controlled trials (RCTs), prospective and retrospective cohort studies, case-control studies, controlled before-and-after studies, historically controlled studies, case series, and interrupted time-series studies. Abstracts and conference proceedings will also be considered.

Case studies, commentaries, editorials, review articles, animal studies and basic science studies, crosssectional studies, as well as studies not reporting relapse/recurrence rates and/or whether consolidation chemotherapy was delivered will be excluded. In studies also including women with other types of GTN (eg, nonmolar, high risk), only data for the postmolar low-risk subgroup will be extracted. To ensure literature saturation, the reference lists of the studies included will be hand searched.

There will be no restrictions on date of publication, geographical location, study setting or language of publication.

\section{Search strategy}

The specific search strategies will be created by a Health Sciences Librarian with expertise in systematic review searching with input from the project team. Searches will be conducted in PubMed (MEDLINE), EMBASE, Web of Sciences, Scopus, LILACS and Cochrane Central Register of Controlled Trials (CENTRAL). A draft search strategy for all databases to be used is presented in online supplemental file 2. After the PubMed strategy is finalised, it will be adapted to the syntax and subject headings of the other databases. Reference lists of included studies identified through the search will be scanned to identify any potentially eligible studies. The search will be updated towards the end of the review to capture recently published literature. 


\section{Data management}

Two independent reviewers will perform data abstraction and quality assessment. Disagreements will be resolved through discussion and/or with the involvement of third-party arbitration. Using standardised forms, the reviewers will extract data independently and in duplicate from each eligible study. To ensure consistency across reviewers, calibration exercises will be conducted before starting the review. The Grading of Recommendations, Assessment, Development and Evaluation Profiler (GRADEpro) software will be used to create tables for summary of findings and quality assessment.

\section{Screening}

All screening steps will be conducted independently by two reviewers. Duplicate references will be removed, and two review authors will independently screen the titles and abstracts yielded by the search. Full-text articles that meet the inclusion criteria will then be retrieved and screened. The reasons for excluding trials will be recorded. Neither of the review authors will be blind to the journal titles or to the study authors or institutions.

To facilitate collaboration among reviewers, search results will be uploaded to a web-based systematic review tool (eg, Rayyan** or COVIDENCE) during the study selection. Training will be provided to members of the review team not familiar with such tools. Prior to the formal screening process, screening questions and forms will be developed and tested for assessments based on the inclusion and exclusion criteria.

The reviewers will meet after each stage to assess agreement. Disagreements will be resolved through discussion. If the conflict persists, a third independent reviewer will be consulted to reach unanimity.

The PRISMA flow diagram ${ }^{10}$ will be used to report the screening process.

\section{Data abstraction}

A data abstraction form will be created and piloted by two reviewers on a sample subset of publications for this review. Data to be collected include study title, authors, publication date, number of patients with postmolar low-risk GTN, lowrisk GTN definition, type of antecedent pregnancy, pretreatment hCG, single-agent chemotherapy regimen used, number of single-agent chemotherapy cycles needed to achieve hCG normalisation, interval between chemotherapy cycles until hCG remission, number of consolidation chemotherapy cycles administered, consolidation chemotherapy adverse events, relapse/recurrence rate, relapse/recurrence definition, time to relapse/recurrence, relapse/recurrence prognostic factors identified.

\section{Quality assessment}

The assessments of RCTs will be performed using the risk of bias tool from the Cochrane Collaboration, ${ }^{11}$ which evaluates potential bias for seven items across six domains: selection bias (random sequence generation; allocation concealment), performance bias (blinding of participants and personnel), detection bias (blinding of outcome assessment), attrition bias (incomplete outcome data), reporting bias (selective reporting), and other sources of bias. Each study will be rated as 'high,' 'unclear' or 'low' risk of bias.

Non-randomised studies will be assessed using the Newcastle-Ottawa scale ${ }^{12}$ that evaluates each study on eight items falling into three categories: (1) selection of study groups, (2) comparability of groups and (3) ascertainment of exposure or outcome of interest.

The quality of evidence will be assessed using the Grading Quality of Evidence and Strength of Recommendations (GRADE) guidelines, ${ }^{13}$ which covers risk of bias, inconsistency, indirectness, imprecision and publication bias. The overall quality of evidence is rated as high, moderate, low or very low by each outcome measure.

The GRADEpro software will be used to create tables for summary of findings and quality assessment. ${ }^{14}$

\section{Data analysis}

Aggregate data will be used, and quantitative synthesis planned where possible if the studies included are sufficiently homogeneous in terms of design and disease type or outcome definitions (homogeneous outcomes in at least two studies). Continuous data will be expressed as means and SD. For dichotomous data, the OR will be calculated, and a log-rank approach will be used to estimate a HR, both with $95 \%$ CI. Random effects is the model chosen for metaanalysis as treatment effect is assumed to vary among studies. For quantitative synthesis, Review Manager V.5.3 software will be used to pool the results of trials for each outcome.

Inconsistency among the results of the included studies will be ascertained by visual inspection of a forest plot (absence of overlap of CIs around the effect estimates of individual studies), as well as by the Higgins inconsistency test or $\mathrm{I}^{2}$, where $\mathrm{I}^{2}>50 \%$ indicates moderate likelihood of heterogeneity.

The quality of evidence of intervention effect estimation for outcomes that can be plotted in meta-analysis will be generated according to GRADE.

Qualitative synthesis is planned for outcomes in which quantitative synthesis is not feasible or appropriate.

Any amendments to this protocol will be documented with their corresponding rationale in the full review.

\section{Patient and public involvement}

There is no patient and public involvement in this study.

\section{Author affiliations}

${ }^{1}$ Botucatu Medical School, Postgraduation Program in Tocogynecology, Sao Paulo State University Julio de Mesquita Filho, Sao Paulo, Brazil

${ }^{2}$ Department of Gynecology and Obstetrics, Botucatu Medical School, Sao Paulo State University Julio de Mesquita Filho, Sao Paulo, Brazil

${ }^{3}$ Botucatu Trophoblastic Disease Center, Botucatu Medical School Hospital, São Paulo State University Julio de Mesquita Filho - UNESP, Botucatu, Brazil ${ }^{4}$ Department of Obstetrics, Gynecology and Reproductive Biology, Harvard Medical School, Boston, Massachusetts, USA

AcknowledgmentsThe authors wish to thank Marluci Bertini, Botucat Medical School Library, for her time and expertise contributed to this protocol, and acknowledge the support of the Donald P. Goldstein MD Tumor Registry Endowment and the Dyett Family Trophoblastic Disease Research and Registry Endowment.

Contributors MB-S will serve as the first author of the protocol and review paper. She led all stages of protocol development, including development of the research question and objectives, search strategy, and extraction and analysis plans. IM, KE, 
RSB, JFA and NSH supervised and contributed to the plans for searching, screening, extracting and writing phases. All authors read and approved the final manuscript.

Funding The authors have not declared a specific grant for this research from any funding agency in the public, commercial or not-for-profit sectors.

Competing interests The authors declare no conflicts of interest.

Patient consent for publication Not applicable.

Provenance and peer review Not commissioned; externally peer reviewed.

Supplemental material This content has been supplied by the author(s). It has not been vetted by BMJ Publishing Group Limited (BMJ) and may not have been peer-reviewed. Any opinions or recommendations discussed are solely those of the author(s) and are not endorsed by BMJ. BMJ disclaims all liability and responsibility arising from any reliance placed on the content. Where the content includes any translated material, BMJ does not warrant the accuracy and reliability of the translations (including but not limited to local regulations, clinical guidelines, terminology, drug names and drug dosages), and is not responsible for any error and/or omissions arising from translation and adaptation or otherwise.

Open access This is an open access article distributed in accordance with the Creative Commons Attribution Non Commercial (CC BY-NC 4.0) license, which permits others to distribute, remix, adapt, build upon this work non-commercially, and license their derivative works on different terms, provided the original work is properly cited, appropriate credit is given, any changes made indicated, and the use is non-commercial. See: http://creativecommons.org/licenses/by-nc/4.0/.

ORCID iD

Mariza Branco-Silva http://orcid.org/0000-0001-8145-2962

\section{REFERENCES}

1 Berkowitz RS, Goldstein DP. Current advances in the management of gestational trophoblastic disease. Gynecol Oncol 2013;128:3-5. doi:10.1016/j.ygyno.2012.07.116

2 Berkowitz RS, Goldstein DP HN. Gestational trophoblastic neoplasia: epidemiology, clinical features, diagnosis, staging, and risk stratification, 2019. UpToDate. Available: https://www.uptodate.com/ contents/gestational-trophoblastic-neoplasia-epidemiology-clinicalfeatures-diagnosis-staging-and-risk-stratification [Accessed 31 Aug 2019].

3 Horowitz NS, Goldstein DP, Berkowitz RS. Management of gestational trophoblastic neoplasia. Semin Oncol 2009;36:181-9.

4 Goldstein DP, Berkowitz RS, Horowitz NS. Optimal management of low-risk gestational trophoblastic neoplasia. Expert Rev Anticancer Ther 2015;15:1293-304.

5 Lawrie TA, Alazzam Mo'iad, Tidy J, et al. First-Line chemotherapy in low-risk gestational trophoblastic neoplasia. Cochrane Database Syst Rev 2016;19. doi:10.1002/14651858.CD007102.pub4

6 Yang J, Xiang Y, Wan X, et al. Recurrent gestational trophoblastic tumor: management and risk factors for recurrence. Gynecol Oncol 2006;103:587-90.

7 Sun Y, Xiang Y, Wan X-run, et al. [Factors related to recurrence of choriocarcinoma and evaluation of treatment outcomes]. Zhonghua Fu Chan Ke Za Zhi 2006;41.

8 Lybol C, Sweep FCGJ, Harvey R, et al. Relapse rates after two versus three consolidation courses of methotrexate in the treatment of low-risk gestational trophoblastic neoplasia. Gynecol Oncol 2012;125:576-9. doi:10.1016/j.ygyno.2012.03.003

9 Couder F, Massardier J, You B, et al. Predictive factors of relapse in low-risk gestational trophoblastic neoplasia patients successfully treated with methotrexate alone. Am J Obstet Gynecol 2016;215:80. e1-80.e7. doi:10.1016/j.ajog.2016.01.183

10 Shamseer L, Moher D, Clarke M, et al. Preferred reporting items for systematic review and meta-analysis protocols (PRISMA-P) 2015: elaboration and explanation. BMJ 2015;349:g7647. doi:10.1136/bmj. g7647

11 Higgins JPT. Cochrane Handbook for systematic reviews of interventions version 5.1.0. The Cochrane collaboration, 2011.

12 Wells G, Shea B, O'Connell D. The Newcastle-Ottawa scale (NOS) for assessing the quality of nonrandomised studies in meta-analyses. Ottawa, ON: Ottawa Hospital Research Institute, 2000.

13 Grade Handbook, 2021. Available: https://gdt.gradepro.org/app/ handbook/handbook.html [Accessed 01 Nov 2021].

14 GRADEpro. Grade your evidence and improve your Guideline development in health care, 2021. Available: https://gradepro.org/ [Accessed 01 Nov 2021]. 